\title{
LA REFORMA DEL TRIBUNAL GENERAL DE LA UNIÓN EUROPEA
}

\author{
VLAD CONSTANTINESCO \\ Catedrático emérito \\ Universidad de Strasbourg
}

«Il est vrai que, par une bizarrerie qui vient plutôt de la nature que de l'esprit des hommes, il est quelquefois nécessaire de changer certaines lois. Mais le cas est rare, et, lorsqu'il arrive, il n'y faut toucher que d'une main tremblante : on y doit observer tant de solennités et apporter tant de précautions que le peuple en conclue naturellement que les lois sont bien saintes, puisqu'il faut tant de formalités pour les abroger» ${ }^{1}$.

\section{SUMARIO}

I. Desarrollo de la reforma. II. Valoración de la reforma.

La reciente reforma del Tribunal General de la Unión Europea por parte del Reglamento 2015/2422 del Parlamento Europeo y del Consejo del 16 de noviembre de $2015^{2}$ que modifica el Protocolo n. 3 sobre el Estatuto del Tribunal de Justicia de la Unión Europea empieza a despertar interés en la doctrina ${ }^{3}$, y también suscita bastantes críticas por la manera en la cual se condujo la reforma, su

1 Montesquieu: Lettres persanes (1721), Lettre LXXIX, de Usbek à Rhédi, à Venise.

2 Del 24.12.2015, DO n. ${ }^{\circ}$ L 341/14.

3 Véase R. Rousselot: «Tribunal: une réforme du statut de la Cour en demi-teinte», Carnets européens. Droit et intégration en Europe, 2016, http://europeanpapers.eu/fr/europeanforum/tribunal-une-reforme-du-statut-de-la-cjue-en-demi-teinte. N. Bermejo Guttierez: «La reforma del Tribunal General de la Unión europea», Almacén de derecho, 17 de mayo 2016, http://almacendederecho.org/la-reforma-del-tribunal-general-dela-union-europea/. A. Huyue ZhANG: «The Faceless Court», Forthcoming in : 38 University of Pennsylvania Journal of International Law (2016), http://dx.doi.org/10.2139/ssrn.2640861. M. VAN DER WoudE: In favour an effective judicial protection: A reminder of the 1988 objectives of the General Court of the EU, Revue Concurrences n. ${ }^{\circ}$ 4-2014, p. 9, http://www.concurrences.com/revue/numeros/no-4-2014/in-favour-aneffective-judicial-protection-a-reminder-of-the-1988-objectives-of. 
coste financiero, y finalmente la impresión que la reforma no llegara verdaderamente a alcanzar sus objetivos ${ }^{4}$.

El sistema judicial de la Unión Europea constaba, antes de la reforma, de tres entidades: 1) el Tribunal de Justicia de la Unión europea (TJUE), 2) el Tribunal General (TGUE, ex Tribunal de Primera Instancia), y 3) el Tribunal de la Función Publica (TFP). El primero apareció cuando fueron redactados y puestos en marcha los tratados creando la CECA, y, de las CEE (Convenio sobre ciertas instituciones comunes). Inicialmente, un solo Tribunal (Tribunal de Justicia de las Comunidades europeas) estaba previsto por los tratados constitutivos. Con el Tratado de Lisboa (2009), el reconocimiento de la personalidad jurídica a la Unión europea, y la desaparición de la «Comunidad europea» como marco general de la integración, cambio su denominación a favor de la de: Tribunal de Justicia de la Unión europea.

Sin embargo, desde el Acta Único Europea (1988) se planteó la necesidad de ampliar la jurisdicción comunitaria, debido al incremento cuantitativo de los distintos recursos. Se impuso entonces la idea de crear un nuevo Tribunal, para descargar el Tribunal de Justicia llamado inicialmente Tribunal de Primera instancia; este Tribunal tiene como base jurídica una decisión del Consejo del 24 de diciembre de 1988. ${ }^{5}$ Recibió su nombre de "Tribunal General» ${ }^{6}$ (en francés, le Tribunal) con el tratado de Lisboa (2007). Es este nivel de la jurisdicción de la Unión al que se refiere la reforma.

Pocos años después, en 2004, fue establecido — por razones similares- el Tribunal de la Función Publica Europea, encargado de solucionar recursos vinculados a la función pública europea, un contencioso importante, por cierto, pero bastante peculiar por las reglas y los principios que se aplican, y además, bastante repetitivo. Esta última jurisdicción permitió aliviar a su vez las cargas de trabajo del Tribunal de Justicia y del Tribunal General. Además, la creación del TFPE

4 El Egmont Paper 83, redactado por Franklin DeHousse (ex juez del Tribunal general) con la colaboración de B. MARsicola, e intitulado: The Reform of the EU Court (II). Abandoning the Management Approach by Doubling the General Court, March 2016, http://www.tepsa.eu/wp-content/uploads/2016/03/paper.pdf contiene un análisis detallado de las distintas fases de la reforma.

Muy bien documentado, muy detallado, este informe de 88 paginas, que presenta la ventaja de haber estado escrito por alguien que conoció el interior mismo del Tribunal General y que pudo seguir paso a paso el desarrollo de su reforma, se muestra critico tanto hacia el contenido mismo de la reforma que hacia la manera de realizarla. Seguiremos en este articulo los principales hallazgos de este documento, en la ausencia, a nuestro conocimiento, de un punto de vista que sea contrario y positivo. El mismo punto de vista critico hacia la reforma es también defendido por el informado periodista Jean QuATremer, en su «blog» Coulisses d'Europe», http://bruxelles.blogs.liberation.fr/2015/04/07/cour-de-justice-europeenne-comment-creer-une-usine-gaz/

5 DOCE, L 319, del 25 de noviembre 1988.

6 Según la doctrina, «(...) el nombre de «General» subraya el carácter de órgano jurisdiccional común que se le atribuye al Tribunal General como jurisdicción de derecho común competente para conocer de todos los recursos salvo aquellos que estén atribuidos al Tribunal de Justicia o a los Tribunales especializados.» R. Garcia Valdecasas Fernandez: Aspectos orgánicos y funcionamiento del Tribunal General y de los Tribunales especializados. Relaciones entre si y con el Tribunal de Justicia, in J. M. . Beneyto Perez (Director), J. Maillo Gonzalez-Orus y B. Becerril Atienza (Coords.): Tratado de Derecho y Políticas de la Unión Europea, tomo V, Sistema Jurisdiccional de la UE, Ed Aranzadi, 2013, p. 155, esp. p. 156. 
corresponde al art. 257. 1 del TF UE que prevé la posibilidad de establecer tribunales especializados juntos al Tribunal General, para aliviar la carga de trabajo de este último.

Este esquema ha sido trastocado por la reciente reforma de 2015-2016 que pone fin a la existencia del Tribunal de la función pública europea e incrementa, de un modo espectacular — ise duplican, es decir 56 jueces!_-, el número de los jueces del Tribunal general, el cual recupera las atribuciones del Tribunal de la Función Pública Europea

\section{EL DESARROLLO DE LA REFORMA ${ }^{7}$}

La reforma se inició conforme a lo dispuesto por el artículo 281 del TFUE ${ }^{8}$ tal como quedó modificado tras el tratado de Lisboa, permitiendo al Tribunal de Justicia iniciar un procedimiento legislativo ordinario destinado a enmendar las disposiciones del Estatuto del Tribunal de Justicia, salvo ciertas excepciones previstas por el mismo artículo. Este cambio, esta apertura del derecho de iniciativa al TJUE, así como el favorecimiento del procedimiento decisional es una de las claves de la reforma. La primera propuesta del TJUE, con fecha del 29 de marzo de 2011, contenía por cierto otros aspectos, pero el que parece relevante fue la demanda de aumentar el numero de jueces el Tribunal General hasta 12, demanda justificada por el incremento continuo del número de asuntos pendientes ante esa jurisdicción, así como sus efectos sobre la duración de los procesos, situación poco compatible con las exigencias del art. 6 \$ 1 del CEDH, y también con las del art. 47 de la Carta de los Derechos Fundamentales de la Unión Europea. La otra posibilidad, la de crear una nueva jurisdicción, especializada y adjunta al Tribunal, permitida por el art. $257 \mathrm{~T}$ FUE, fue finalmente rechazada.

Contestando a una demanda del Consejo, el Tribunal estimaba en algo más de 13 mil millones de euros el impacto presupuestario de la reforma propuesta. En setiembre del mismo año, la Comisión aprobó la demanda de Tribunal $^{9}$, subrayando algunas precauciones que deberían tomarse para llevar a cabo la reforma, por ejemplo en creando cámaras especializadas en el seno mismo del TG (lo que es finalmente una manera indirecta de crear jurisdicciones especializadas),

7 Para un estudio detallado de todas las etapas de la reforma se consultara el informe precitado de $\mathrm{Fr}$. DeHOUSSE.

8 El Tribunal disponía antes de este derecho de iniciativa (art. 188 T CE, revisado por el T de Maastricht, art. $245 \mathrm{~T}$ CE, revisado por el T de Niza) pero las reglas de modificación del Estatuto eran las de la revisión de los tratados, sin participación del Parlamento europeo y con la unanimidad des los Estados miembros. El T de Lisboa introdujo esta iniciativa de TJUE en el marco del procedimiento legislativo ordinario, es decir con participación del PE y voto del Consejo a la mayoría cualificada. Si, antes, cada uno de los Estados miembros podía proponer enmiendas, en el nuevo marco des T FUE, el derecho de iniciativa solo pertenece al TJUE o a la Comisión.

9 COM (2011)596 final. 
y asegurando la estabilidad del TG, a pesar del incremento espectacular del número de sus jueces.

El Parlamento Europeo reaccionó por primera vez con el informe de la euro-diputada Alexandra Thein ${ }^{10}$, del 17 de julio de 2013, apuntando que el Tribunal General ya había reaccionado frente a los asuntos en demora. Sin embargo, consideraba que había que insistir en la reforma, teniendo precaución con la elección de nuevos jueces que tendrían que tener bueno conocimiento del derecho de la Unión, y también representar a los distintos sistemas jurídicos presentes en los Estados miembros.

En 2012, los Estados miembros, sin embargo, no pudieron ponerse de acuerdo sobre el número de jueces que habría que nombrar. La nueva propuesta del Tribunal de Justicia, de octubre 2014, fue la de doblar el número de jueces del Tribunal General, de una manera escalonada en el tiempo: un aumento de 12 jueces en 2015, de 7 en 2016, y de 9 en 2019. Tanto el Consejo como en el Tribunal de Justicia, sin embargo, se pudo percibir que el TG ya había, en ese momento, puesto al corriente los asuntos pendientes, lo que ponía en duda la pertinencia y la utilidad de la reforma prevista, a pesar de los argumentos — discutibles - avanzados por el Consejo sobre el coste de la no-reforma. ${ }^{11}$

El debate en el Parlamento Europeo acerca de la nueva toma de posición del Consejo en primera lectura adoptada el 23 de junio $2015^{12}$ fue mucho mas intenso que en 2013. En efecto, el informe del euro-diputado Marinho e Pinto respecto a la Posición del Consejo en primera lectura con vistas a la adopción del Reglamento del Parlamento Europeo y del Consejo por el que se modifica el Protocolo n. 3 sobre el Estatuto del Tribunal de Justicia de la Unión Europea ${ }^{13}$ llega a conclusiones severas y al mismo tiempo, razonables y, à nuestro juicio, equilibradas:

«1. Rechazo de la propuesta de duplicar el número de jueces del TG y de suprimir el Tribunal de la Función Pública y, de conformidad con el artículo 69, apartado 2, letras a), e) y d), restablecimiento de la posición aprobada por el Parlamento en primera lectura, debiendo el Tribunal de Justicia justificar el número exacto de jueces efectivamente necesarios, teniendo en cuenta los cambios que se produzcan en las circunstancias, en particular el cambio de tendencia en relación con el número de asuntos nuevos y de asuntos resueltos.

10 Informe, en nombre de la Comisión de Asuntos jurídicos, sobre el proyecto de Reglamento del Parlamento Europeo y del Consejo por el que se modifica el Protocolo sobre el Estatuto del Tribunal de Justicia de la Unión Europea para aumentar el número de Jueces del Tribunal General (02074/2011 — C7-0126/2012 - 2011/0901B(COD)).

11 El Consejo avanzaba la enorme suma de 23 mil millones de EURO, basada, curiosamente, en el hecho que distintas compañías habían estimado a esta cifra la compensación que podrían obtener por violación de la obligación de ver sus casos juzgados en un plazo razonable...

12 Según Fr. Fdehousse (op. cit., p. 30) el Reino-Unido voto en contra, mientras Bélgica y Países-Bajos se abstuvieron.

$13(09375 / 1 / 2015$ - C8-0166/2015 - 2011/0901B(COD)). 
2. Rechazo de la propuesta de supresión del TFP por carecer de fundamento jurídico en el Tratado, debiéndose proceder de inmediato al nombramiento de los jueces ya designados y a la designación de los que faltan; creación de ulna comisión de expertos para analizar las ventajas y los inconvenientes de la creación de un nuevo tribunal especializado en marcas, patentes y propiedad intelectual.

3. De conformidad con el Reglamento del Parlamento Europeo, artículo 69, apartado 2, letra c), se recomienda el nombramiento de diecinueve letrados de modo que cada juez disponga de un letrado más (teniendo en cuenta que ya se nombraron nueve en 2014), solución ya adoptada en el Tribunal de Justicia. Se recomienda asimismo un aumento de los funcionarios adscritos a la Secretaría y los servicios de traducción.

4. Se recomienda la creación, por parte del Parlamento y del Consejo, de una comisión conjunta de expertos para examinar el funcionamiento global de la justicia en la Unión y presentar propuestas de mejora, teniendo en cuenta, entre otros, los siguientes aspectos:

a) Selección de los jueces a través de un concurso abierto entre profesores de Derecho de reconocido mérito y entre magistrados de los tribunales superiores de cada uno de los Estados miembros;

b) Designación de cada juez para un único mandato de nueve años, sin posibilidad de renovación o ampliación. (...)»

La mayoría de los euro-diputados discrepó de esta opinión. El voto final del Parlamento tuvo lugar el 28 de octubre 2015, aceptando la Posición del Consejo en segunda lectura, sin aceptar las críticas y los argumentos del ponente, el cual, y de manera bastante excepcional y enigmática, solicitó:

«Senhor Presidente, Caros Colegas, é só para dizer que quero retirar o meu nome deste relatório porque não é o relatório que eu fiz. Quem fez este relatório que o subscreva, que não eu» ${ }^{14}$.

\section{VALORACIÓN DE LA REFORMA}

La manera en que la reforma se desarrolló — por voluntad del anterior presidente del Tribunal de Justicia y contra la opinión del mismo presidente del Tribunal General $^{15}$ y la del ponente Marinho e Pinto_- suscita reservas, y quizás criticas ${ }^{16}$.

La reforma no solo modifica el esquema general de la justicia de la Unión europea, haciendo desaparecer el Tribunal de la Función Publica, sino también la

14 http://www.europarl.europa.eu/sides/getDoc.do?type $=$ CRE\&reference $=20151028 \&$ secondRef $=$ ITEM-007-03\&language $=\mathrm{ES} \& \mathrm{ring}=\mathrm{A} 8-2015-0296$

15 Ver detalles en el informe precitado de Fr. DeHousse.

16 Reenvío una nueva vez al informe precitado de Fr. Dehousse, que contiene abundantes detalles sobre las distintas peripecias que llevaron a la reforma, y también en su 5. ${ }^{\text {a }}$ parte, explicaciones sobre: «Lessons for judicial Management», y en su 6. ${ }^{a}$ parte: «Lessons for the Exercise of the Court's legislative Power». 
arquitectura misma del aparato jurisdiccional de la Unión. No es preciso recordar aquí el papel eminente que jugo - y que todavía despliega - el Tribunal de Justicia de la Unión europea (TJUE) en el desarrollo y en la dinámica del derecho de la Unión. Su competencia en interpretación y aplicación de los tratados constitutivos (derecho originario) - y también del derecho derivado- le confiere una autoridad central en un proceso de integración que se fundamenta en la regla jurídica aceptada tanto por los Estados miembros como por los operadores económicos, y, más allá, por los ciudadanos de la Unión. Se ha repetido, no sin razón, que la interpretación y la aplicación correcta de la regla de derecho fue y sigue siendo el vector esencial por el cual pasan las decisiones políticas tomadas por las instituciones encargadas por los tratados de elaborar las normas del derecho derivado.

La estructura anterior, con sus tres niveles y con su especialización, permitía un funcionamiento correcto del sistema jurisdiccional. ¿A partir del momento en el cual los datos que justificaban la reforma se revelan inexactos - el supuesto retraso del TG que se recuperó- uno puede preguntarse si un TG con 56 jueces es una solución convincente? ¿ En particular, cuál podrá ser la carga de trabajo de cada juez? ¿No habrá demasiados jueces en el futuro en el TG? Otras preguntas, como el coste de la reforma o la calificación de los futuros jueces quedan abiertas. También uno puede lamentar la desaparición del TFPUE: un contencioso de ámbito limitado que tiene toda su peculiaridad ahora en manos de jueces que no estarán necesariamente al tanto de esta especialidad. Tal decisión deja al margen las soluciones de jurisdicciones especializadas, en particular para el contencioso de propiedad intelectual y de marcas, otro contencioso especializado que necesitaría su propio tribunal, aliviando el TG.

Esta reforma cuestiona también la legitimidad del juez de la Unión, la cual, como se sabe es, a veces, cuestionada con el reproche de faltar de legitimidad democrática, reproche que muchos dirigen, de manera general, a todas las instituciones de la Unión ${ }^{17}$. Como se sabe, la legitimidad de una jurisdicción reside en una multitud de criterios, entre los que el principio democrático no es el más relevante. La legitimidad peculiar de una jurisdicción se mide sobre todo en la apertura de su foro, la calidad de su jurisprudencia, la motivación de sus decisiones, el plazo razonable en el cual la jurisdicción resuelve el caso que se le plantea; calcularla en términos de legitimidad democrática poco tiene que ver con esos criterios. Pero la reforma pone sobre todo en duda la capacidad de iniciativa del mismo Tribunal de Justicia, lo que se refleja en su legitimidad. De la opinión de muchos exégetas se ha podido notar la falta de una estrategia global, a largo plazo, permitiendo controlar — con ayuda de expertos y transparencia- todos los datos y requisitos para llegar a una reforma funcional.

17 Cf. D. Simon: «La légitimité du juge communautaire», Paris, Palais du Luxembourg (Colloque sur L'office du juge, tenu au Sénat les 29 et 30 septembre 2006), https://www.senat.fr/colloques/office_du_juge/ office_du_juge42.html. 
Mas allá de ello, cabe cuestionar si debe pertenecer a la principal jurisdicción de la Unión europea la capacidad de iniciar un procedimiento legislativo. Está claro que el sistema institucional de la Unión no responde exactamente la la «separación de poderes», a la «trias política», establecida por Montesquieu en su obra L'esprit des lois.

Ya se sabe que la distribución de poderes en el ordenamiento institucional de la UE ni corresponde ni obedece a los parámetros distinguidos por Montesquieu $^{18}$. En realidad, es una lógica de representación de los intereses que explica la composición y los poderes de cada institución, como los equilibrios que realizan los procedimientos decisionales. Una misma función - como por ejemplo la función normativa - estará ejercitada por medio de distintas instituciones. Y una misma institución ejercitara poderes de distintas naturalezas. En otros términos, a la separación orgánica de los poderes no corresponde una separación funcional. Y la cosa se complica si añadimos las distintas instituciones que al nivel nacional, participan al funcionamiento institucional de la Unión.

Sin embargo, a pesar de la grandes diferencias entre el nivel nacional y el europeo, una máxima de Montesquieu no deja de ser útil: la necesidad de diferenciar entre juzgar la conformidad a y hacerla —iniciarla - o ejecutarla. El art. 281 TFUE se puede leer como un intento — logrado- de escapar la revisión de los tratados y al costoso y difícil procedimiento del art. $48 \mathrm{~T}$ UE, por medio del simple procedimiento legislativo, dando la exclusividad de la iniciativa al Tribunal de Justicia, en una materia tan importante como la modificación de su Estatuto. Se sabe que un principio fundamental en el ámbito jurídico de la Unión europea, es el que permite distinguir las normas de nivel constitucional de las de nivel inferior. El art. 281 TF UE, aunque establecido por los Estados en el marco de un procedimiento de revisión, no parece haber respetado esta distinción. Además, una institución como el Tribunal de Justicia esta demasiado cerca de sí misma para poder tener el necesario retroceso para tomar en cuenta - con el máximo de imparcialidad - todos los elementos involucrados en una reforma de tal tamaño. Se puede recordar el precepto antiguo del derecho romano: «Nemo judex in causa sua». Tampoco tendrá el Tribunal de Justicia la capacidad de negociar con otras instituciones, lo que es una de las claves del éxito del procedimiento de codecisión.

En esta materia no hay razón ni duda para no restablecer, en una futura reforma, la iniciativa de la Comisión. Que el Tribunal de Justicia pueda provocar una iniciativa de la Comisión parece ser una solución adecuada y razonable. El mantenimiento de la normativa en vigor no es una garantía — itodo lo contrario! - del éxito de futuras reformas que el sistema jurisdiccional de la Unión tendrá de todos modos que enfrentar en el futuro. Al contrario, el precedente examinado aquí demuestra la necesidad de cambiar las reglas del juego, para el

18 L'Esprit des lois, (1748) Libro VI, capitulo XI. 
mejor funcionamiento del sistema jurisdiccional, respetando la lógica de la diferencia entre revisión constitucional y procedimiento legislativo y las responsabilidades propias de cada institución.

TITLE: The reform of the General Court of the European Union.

ABSTRACT: The recent reform of the General Court of the European Union by the 2015 regulation / 2422 of the European Parliament and of the Council of 16 November 2015 is worthy of special attention. It is likely to be criticized from various points of view: at least for the way in which led reform, financial cost, and finally the impression that reform did not come truly to achieve their goals. This article explains the scope of reform and various criticisms that can be performed to it.

Resumen: La reciente reforma del Tribunal General de la Unión Europea por parte del Reglamento 2015/2422 del Parlamento Europeo y del Consejo del 16 de noviembre de 2015 es digna de especial atención. Es susceptible de ser criticada desde diversos puntos de vista: al menos por la manera en la cual se condujo la reforma, su coste financiero, y finalmente la impresión que la reforma no llegara verdaderamente a alcanzar sus objetivos. Este artículo explica el alcance de la reforma y las diferentes críticas que cabe realizar a la misma.

KEY WORDS: General Court of the European Union, financial cost, number of judges.

Palabras Clave: Tribunal General de la Unión Europea, coste financiero, número de jueces.

FECHA DE RECEPCIÓN: 15.01.2017

FECHA DE ACEPTACIÓN: 01.02.2017 